Escuela de Ciencias Administrativas, Contables, Económicas y de Negocios
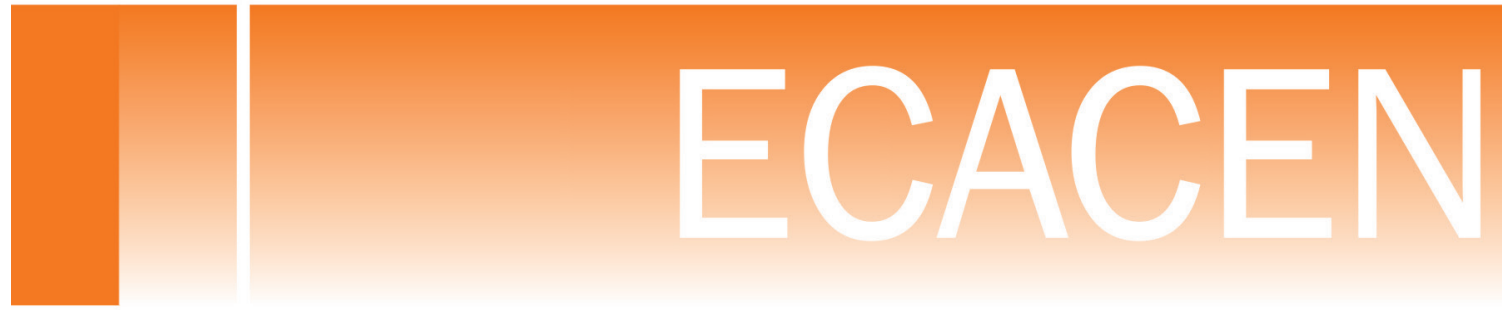



\title{
CARACTERIZACIÓN DE LAS PRÁCTICAS DE RESPONSABILIDAD SOCIAL ORGANIZACIONAL EN LA EMPRESA BOGOTANA COMO HERRAMIENTA PARA EL DESARROLLO FUTURO DE PROGRAMAS DE FORMACIÓN SUPERIOR
}

\author{
Ariel Alfonso Reyes Castro y \\ Luz Stella González Santafée
}

\begin{abstract}
RESUMEN
El proceso de globalización de los mercados demanda el entendimiento estratégico del aporte que genera la responsabilidad social corporativa para desarrollar negocios sostenibles en el largo plazo. Por lo tanto, tal situación propone un campo de investigación en evolución, que tiene su punto de partida en el comportamiento organizacional y la ética empresarial.

De esta manera, esta ponencia mostrará los resultados de una investigación cuantitativa desde una perspectiva descriptiva, para caracterizar las prácticas que las empresas de Bogotá, Colombia, llevan a cabo en materia de responsabilidad social corporativa. En consecuencia, el resultado de este trabajo entrega un amplio rango de conocimiento que permite el fomento y la implementación de lo que es la responsabilidad social en las industrias y negocios de Bogotá. Además, a través de este proceso investigativo, la comunidad académica colombiana será capaz de desarrollar programas de formación basados en los enfoques estratégicos que toman en cuenta la responsabilidad social como parte del sistema llamado empresa, incluyendo las pequeñas y medianas. Por lo tanto, esta investigación estimula a que cada emprendedor tome decisiones para construir una consciencia responsable como respuesta a las demandas de los actores empresariales tanto internos como externos, sin importar el giro de negocio sobre el cual se desarrolla su unidad económica.
\end{abstract}

Palabras clave: responsabilidad social, stakeholders, prácticas, empresas bogotanas, programas de formación.

\begin{abstract}
The globalization process of the markets demands the strategic understanding of the contribution that generates the corporate social responsibility to develop sustainable business in a long term. Therefore, this situation suggests an evolving research field, which has its starting point in organizational behavior and corporative ethics.

Thus this paper will show the results of a quantitative research from a descriptive perspective, to characterize the practices that companies in Bogotá, Colombia, carried out in the field of corporate social responsibility. Consequently, the result of this paper delivers a broad range of knowledge that allows the development and implementation of what is the social
\end{abstract}

$\overline{26}$ Escuela de Ciencias Administrativas, Contables, Económicas y de Negocios, ECACEN, UNAD. 
responsibility in Bogota's companies and business. In addition, through this investigative process, the Colombian academic community will be able to developing training programs based on strategic approaches taking into account social responsibility as part of the system named company, including small and medium enterprises. Therefore, this research encourages every entrepreneur to make decisions to build a responsible consciousness in response to demands of stakeholders both internal and external business, regardless of the turn of business on which it develops its economic unity.

Key words: social responsibility, stakeholders, practices, enterprise, formation programs, economic unity.

\section{INTRODUCCIÓN}

El concepto de responsabilidad en Colombia ha pasado por diferentes vertientes que van del mecenazgo y el patrocinio a la filantropía. Sin embargo, estas concepciones inspiradas en la tradición cristiana del país, no son en realidad prácticas de responsabilidad social. Por ejemplo, empresas como Nike y Parmalat patrocinan equipos de futbol (mecenazgo) y realizan campañas sociales a favor de la niñez (filantropía); no obstante, también han estado involucradas en situaciones asociadas con prácticas laborales abusivas (la primera) y de abuso de información para cometer fraude (la segunda). Así, nos encontramos con un primer problema y corresponde a que no existe un mecanismo claro, para establecer qué prácticas corresponden a modelos de gestión que de manera voluntaria evalúen prácticas de responsabilidad social empresarial (RSE).

También existe otro elemento para caracterizar y consiste en que hoy por hoy la respuesta de las organizaciones que son acusadas por realizar actividades contrarias a la libre competencia, por hacer mal manejo ambiental, por incidir de manera negativa en la sociedad o por tener prácticas laborales contrarias a la ley, no ha sido estratégica ni operativa, sino más bien cosmética puesto que se enfocan en lanzar campañas publicitarias usando como núcleo central un reporte de sostenibilidad que muestra las buenas obras sociales y ambientales de la empresa (Porter, 2006), pero que casi nunca es auditado por terceros, principalmente, porque casi nadie lo sabe hacer.

Por lo tanto, los partidarios de la RSE han establecido que para que las empresas la implementen dentro de su know How, deben hacerlo a partir del cumplimiento de su respectiva obligación moral (no legal), como un mecanismo al que, a partir de ciertas prácticas, se logra 
la sustentabilidad. Se mejora la reputación ${ }^{27}$ y se obtiene por ende una licencia de operación que se traduce en un mejor desempeño en el mercado. Sin embargo, el medir y hacer público el actuar de las firmas, que aunque es una forma eficaz de medir la conducta social corporativa, únicamente es útil cuando los índices utilizados midan de manera consistente, reflejando el impacto social corporativo de la firma con sus grupos de interés. De esta manera, además de los criterios utilizados, se podría hablar del DJSI ${ }^{28}$ y del FTSE4Good ${ }^{29}$ que junto con el MERCO y el Great Place to Work no cumplen con las condiciones citadas et supra.

Por otra parte, si bien es cierto que ninguna empresa sana funciona en un entorno enfermo y que las corporaciones exitosas necesitan de una sociedad sana; también es necesario establecer que la empresa provoca siempre impactos en la sociedad con sus operaciones como mecanismo para el cumplimiento de su objeto social, estableciendo así vínculos de adentro hacia afuera. Así, no sólo la actividad corporativa tiene efectos en la sociedad, sino que también el entorno influye sobre la firma estableciendo un vínculo de afuera hacia adentro. ${ }^{30}$ Por ende, esos impactos inciden en la productividad y competitividad de la firma, influenciando así su actuar por medio del desarrollo de ventajas competitivas utilizando su cadena de valor. De esta manera, desde el punto de vista empresarial es necesario mirar esas interrelaciones y el impacto que estas tienen en la generación de valor de la firma específicamente en lo relacionado con:

Desarrollo de tecnología que garantice:

- Seguridad en los productos

27 Sin embargo, ¿cómo medir dicha reputación? Quizá dos de los mecanismos usados en la actualidad son el great place to work (que evalúa prácticas laborales) y el MERCO (monitor español de reputación corporativa) que evalúa a las empresas con mayor reputación en:

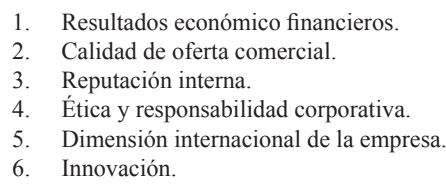

Lo que a la postre se convierte en un ingrediente bien estructurado de la evaluación de un top of mind en donde aparte de las empresas que tradicionalmente se conocen no se incluye ninguna otra diferente.

28 El Dow Jones Sustentable Index incluye aspectos de desempeño económico en su evaluación que pondera el servicio al cliente por encima de las prácticas de ciudadanía corporativa.

29 Este índice no contiene en absoluto indicadores económicos o de servicio al cliente lo que hace que, aunque sus criterios sean similares a los del merco, se obtengan resultados diferentes al final.

30 De esta manera los elementos que establecen la competitividad de una firma cuando se integra a ella el concepto de RSC se pueden dividir en 4 elementos a saber:

1. La cantidad y calidad de los insumos de negocios disponibles, por ejemplo recursos humanos y naturales o infraestructura de transporte.

2. Las reglas e incentivos que rigen la competencia, como las políticas que salvaguardan la propiedad intelectual, garantizan la transparencia, protegen contra la corrupción e incentivan la inversión.

3. El tamaño y sofisticación de la demanda local, influida por asuntos como los estándares de calidad y seguridad de producto, los derechos de los consumidores y la equidad en las compras del gobierno.

4. La disponibilidad local de industrias de apoyo, tales como productores de maquinaria y proveedores de servicios. 
- Conservación de materias primas

- Reúso, reutilización y reciclaje de materias primas e insumos

- Relaciones con universidades y centros de investigación

Elementos ligados a la infraestructura de la empresa que garanticen:

- Prácticas de buen gobierno corporativo

- Transparencia

- Educación y capacitación laboral.

- Condiciones laborales seguras

- Diversidad y no discriminación

- Salud y otros beneficios ligados a la compensación

- Políticas de despido

En lo relacionado con abastecimiento:

- Cuidado y control de recursos naturales.

- Prácticas de abastecimiento y SCM enfocadas a evitar dumping social, trabajo infantil o soborno.

En lo relacionado a actividades primarias es necesario hacer énfasis en:

- Lo relacionado con logística de entrada y su impacto en el transporte (huella ecológica, emisiones, rutas, etcétera).

- En lo relacionado con operaciones de la firma será necesario considerar: emisiones, uso de recursos, seguridad y relaciones laborales, manejo de riesgos laborales.

- En la logística de salida ser necesario hacer énfasis en el manejo de la logística inversa (en lo relacionado con el e-waste, por ejemplo).

- En cuanto a marketing será clave enfocarse en el concepto de marketing social, la publicidad veraz, las prácticas de fijación de precios y el impacto que el dumping social y ecológico tiene para la competitividad de la firma.

- En cuanto al servicio posventa será clave tener en cuenta elementos asociados a información al consumidor, privacidad y todo lo relacionado con mercadeo y servicio.

Sin embargo, a raíz de lo anterior, surge un problema: si las empresas saben lo que deben hacer, ¿por qué no lo ponen en la práctica?, ¿Será que no han tomado en cuenta el impacto que tiene en su competitividad o será que no tienen la menor idea de cómo implementarlo en su organización? De esta manera queda claro que para que las organizaciones puedan resolver adecuadamente los problemas que se mencionaron en este acápite, es necesario integrar a 
la academia, que como ente con dos propósitos ${ }^{31}$ claramente definidos, puede ayudar en la caracterización de modelos de gestión que minimicen el impacto de la actuaciones endógenas y exógenas que adelanta la firma Vallaeys Francois (2007).

Consecuentemente, a partir de los impactos de la organización, surgen cuatro ejes de actuación de la academia como garante del desarrollo de modelos de gestión que permitan el desarrollo de prácticas socialmente sostenibles. Los cuales pueden caracterizarse de la manera siguiente:

1. La universidad puede trabajar en la gestión socialmente responsable de la organización a través del desarrollo de modelos y políticas que permitan gestionar recursos humanos, procesos democráticos de autogobierno y cuidado del medio ambiente.

2. La universidad debe trabajar en la gestión socialmente responsable de la formación académica y la pedagogía tanto de sus temáticas, su metodología de enseñanza y su organización curricular.

3. La universidad debe encargarse de diseñar mecanismos que permitan la gestión socialmente responsable de la participación social en el desarrollo humano sustentable de la comunidad con la que interactúa la organización.

4. Finalmente, y a raíz de lo anterior, la academia debe desarrollar modelos que permitan gestionar la producción y difusión del saber, la investigación y los modelos epistemológicos que desde el aula puedan ser aplicados a la organización.

Con esta caracterización, surge un elemento adicional enfocado básicamente a definir qué áreas del saber, así como también en qué habilidades, competencias y destrezas se debe profundizar desde la academia con mayor profundidad; con el fin de desarrollar y aplicar la RSE en la firma y de esta a la sociedad y el entorno, en una relación biunívoca, multidisciplinaria y multilateral. ${ }^{32}$ De esta manera, y con esta somera introducción, es que se hace necesario preguntarse:

¿Cuáles son las prácticas en responsabilidad social corporativa que aplican las empresas bogotanas y que al identificarlas servirán como base para el desarrollo de programas de capacitación en RSC por parte de la academia lograran que las organizaciones logren un desarrollo integral sostenible?

31 Sus propósitos son (formación humana y profesional, propósito académico, y construcción de nuevos conocimientos (propósito de investigación)

En el caso de la academia dichos impactos se enfocan en:

1. Impactos en el funcionamiento organizacional

2. Impactos educativos

3.Impactos cognitivos y epistemológicos

4. Impactos sociales.

32 De ahí que una de las intencionalidades de la presente investigación sea saber qué se está haciendo sobre el tema en la academia bogotana y cómo esto contribuye al desarrollo de modelos de gestión que incremente la productividad en la organización vía desarrollo de procesos socialmente responsables. 
Para poder resolver dicho interrogante también se hace necesario dividir este interrogante en elementos más pequeños que deben ser utilizados para poder responder a cabalidad la pregunta expuesta et supra, los cuales se citan a continuación:

- ¿Cuáles serán las prácticas que adelantan las empresas bogotanas relacionadas con el tema de la responsabilidad social?

- ¿Qué impacto tiene la aplicación de dichas prácticas en aspectos como los que contempla el triple balance?

- ¿Qué mecanismo de medición de impacto tienen dichas prácticas dentro de la gestión empresarial que realizan las firmas?

- ¿Será necesario capacitar a la empresa bogotana en el desarrollo y puesta en marcha de prácticas relacionadas con el concepto de responsabilidad social?

- ¿Qué practicas adelanta la academia para la capacitación de empresarios, emprendedores y sociedad en el desarrollo de modelos de gestión socialmente responsable?

- ¿Bajo la concepción de la gestión del conocimiento qué elementos conceptuales podrían permitir el desarrollo de programas de formación que faciliten el complimiento de la RSU desde la academia hacia el sector real?

De esta manera, el resultado que mostrará la presente ponencia se enfocó en retomar elementos del estado de la cuestión (Principalmente asociados a la encuesta anual que sobre el tema adelanta la asociación nacional de empresarios ANDI), de analizarlos y de establecer una metodología de investigación enfocada a caracterizar unas prácticas previas sobre el tema de investigación, para posteriormente empezar con la realización de una encuesta a empresas caracterizadas de acuerdo con la normatividad vigente en el país ${ }^{33}$ con el fin de cubrir la información faltante.

\section{METODOLOGÍA}

A raíz de los elementos básicos caracterizados en la introducción de esta ponencia, los siguientes fueron los elementos a considerar para el desarrollo holístico de esta investigación que tiene como objetivo ser el sustento básico para el desarrollo de programas de formación sobre el tema, que se sustentó de la siguiente manera:

33 Ley 905 de 2004 artículo 2. 
Tipo de investigación: la metodología utilizada para la realización de la presente ponencia se enmarcó en una primera etapa en una investigación de tipo descriptiva, ${ }^{34}$ en virtud de la información disponible, para luego tras un análisis exhaustivo de dicha información optar por acudir a diversos métodos de investigación como la observación y el análisis de la información de manera cuantitativa, lo que complementó el estado del conocimiento actual sobre el tema mediante la realización de una encuesta de tipo diagnóstico en la que por medio de la observación de las distintas actuaciones de 76 firmas analizadas se pudo completar y deducir la información de la presente investigación.

Métodos y técnicas de investigación utilizados: los métodos y técnicas de investigación utilizados para poder cumplir con los objetivos propuestos en este trabajo de exploración se exponen a continuación.

- En primer lugar, se caracterizaron por medio de la observación y el análisis, las actuaciones de los 3 gradientes de la productividad que según el modelo de la triple hélice (empresa privada, sector público y academia) condicionan la competitividad de una región.

- Para esto se acudió a verificar el impacto que el sector público y privado tenían en lo relacionado con el cumplimiento de los objetivos del milenio, así como también en lo que tiene que ver con los parámetros de evaluación que considera el pacto mundial. Utilizando principalmente información de las Naciones Unidas en lo relacionado con avances en el logro de los ODM y resultados de prácticas de RSE, según diferentes eventos que sobre el tema se realizaron en la ciudad de Cali en el mes de noviembre de 2009 y en la ciudad de Punta del Este (Uruguay) en el mes de diciembre de 2009.

- Asimismo, en relación con la academia se revisaron los respectivos GroupLac en la base de Colciencias, estableciendo así las prácticas y productos que sobre el tema desarrolla la academia colombiana.

- Una vez hecho esto, se procedió a contrastar la información que la ANDI entregó en el análisis de su encuesta de prácticas de RSE en la vigencia 2008-2009, con el fin de establecer áreas de interés, así como también deficiencias en dichas prácticas dentro de las empresas que dicho mecanismo de auditoría analizó.

De esta manera, una vez que la revisión de las fuentes existentes terminó, se establecieron una serie de falencias, razón por la cual se diseñó un instrumento de encuesta para ser aplicado en el diseño muestral tal y como se ilustra más adelante. También, se acudió a revisión

34 Esto en la medida en que con la realización del presente trabajo de campo se espera establecer:

- Las prácticas asociadas a la implementación de programas y/o actividades que permitan caracterizar el know how asociado al desarrollo de comportamientos vinculados al desarrollo de modelos de gestión socialmente responsables.

- Identificar las formas de conducta y actitudes de las organizaciones que se encuentran en el universo de la investigación con respecto al tema de investigación propuesto.

- Definir de manera tangible comportamientos concretos de las organizaciones que se analizaron para la realización del presente trabajo de investigación.

- Describir y comprobar por medio de análisis bi-variado la posible asociación de variables de investigación con el fin de establecer contenidos temáticos que puedan ser aprovechados por la academia para el acompañamiento, formación, y desarrollos futuros sobre el tema. 
bibliográfica en lo relacionado con caracterización de prácticas de responsabilidad social universitaria (Que se mencionan en el acápite de bibliografía de esta investigación ), con el fin de integrar a la universidad en el desarrollo de un modelo de formación que a partir de la caracterización de las prácticas del sector real público y privado pudiera responder de manera sustentable con programas de capacitación que permitan el desarrollo de modelos de gestión que forjen de manera sostenible el know how de las empresas en la ciudad de Bogotá.

Selección de la muestra. Los criterios de selección de la muestra de investigación fueron los siguientes:

- Dadas las limitantes de tiempo y disponibilidad de recursos e información se optó por diseñar una encuesta que cubriera seis elementos básicos que por su flexibilidad permitieran analizar de manera integral las nueve áreas de interés evaluadas por la norma SGE 21:2008.

- A partir de lo anterior, se investigó en la Superintendencia de Sociedades con el fin de establecer el número de empresas formalizadas en la ciudad de Bogotá.

- Se estableció que la forma de recabar la información implica realizar un tipo de muestreo estratificado (en donde claramente cada estrato estaba conformado por un número de empresas tal que representara de manera equitativa a aquellas firmas caracterizadas de acuerdo con el numeral 2 de la ley 905 de 2004).

Dado que el universo no es homogéneo, sino que está formado por estratos diferentes, tipologías de empresas por tamaño que constituyen categorías importantes para la investigación, la elección de la muestra no se hizo de manera global sino que se escogió un tamaño muestral diferente para cada estrato a partir de información suministrada en 2006 por la Cámara de Comercio de Bogotá ( CCB ) que comunicaba que del total de empresas formalmente constituidas se reportaba la siguiente información: Microempresas $(88 \%)$, Pequeñas $(9 \%)$, Medianas (2\%) y Grandes (1\%). De esta manera, para escoger el tamaño muestral de cada estrato se acudió a la técnica de muestreo estratificado asumiendo un tamaño poblacional equivalente a 1.683 .000 empresas a 2008 según Supersociedades. Asimismo, se asumió que el $50 \%$ de las mismas están en la ciudad. Así, el tamaño muestral utilizado será de 841.500 firmas. Se asumirá un porcentaje de error del 0,015 y un nivel de confianza obteniendo así los siguientes resultados:

Error al cuadrado $\left(0,015^{2}\right)=0,000225$

Coeficiente de confiabilidad $(0,95) *(1-0,95)=0,0475$

$N^{\prime}=0,0475 / 0,025=211,11$

Tamaño muestral $n=\frac{n^{\prime}}{1+n^{\prime} / N}$ 
Equivale a: $211,11 /(1+(211,11 / 841.500))=211$ muestras. Distribuidas así:

- Microempresas $=88 \% * 211=186$ muestras.

- Pequeñas empresas $=9 \% * 211=19$ muestras.

- Medianas empresas $=2 \% * 211=4$ muestras.

- Grandes empresas $=1 \% * 211=2$ muestras.

Sin embargo, para cubrir fallas estadísticas se realizaron 264 encuestas de las cuales sólo el $28,78 \%$ de la información reportada (76 encuestas) cumplía al 100\% con la información necesaria para poder cumplir a cabalidad con el objeto social de la presente investigación. El resto de las mismas 188 omitían información (La mayoría Mipymes) o la entregaban incompleta logrando así el siguiente consolidado sobre el cual se trabajó:

- Microempresas 16

- Pequeñas empresas 13

- Medianas empresas 14

- Grandes empresas 33

A partir de esta información es necesario establecer que aunque el tamaño muestral usado en el análisis de las empresas no corresponde con el tamaño muestral calculado, se optó por usar este, con el fin de realizar la investigación con la hipótesis de que las grandes empresas deberían ser las destinatarias de programas de formación y capacitación a futuro ofertados por la academia. Posteriormente y en las conclusiones se pudo validar a cabalidad la hipótesis alterna que se deduce de la citada et supra.

\section{Análisis e interpretación de la información obtenida}

La metodología usada para la realización del análisis e interpretación de la información obtenida fue la siguiente:

- En lo relacionado con prácticas de los tres elementos que componen el modelo de la triple hélice se optó por utilizar un método descriptivo basado en la evaluación de fuentes secundarias que, de manera descriptiva, permitieron la realización de un análisis estructural.

- En lo relacionado con el análisis de la herramienta de diagnóstico se optó por realizar un análisis univariado de la información con el fin de establecer variables clave, analizarlas y establecer una caracterización básica de prácticas de interés para el presente estudio. Posteriormente, con la definición de dichas variables clave se procedió a realizar un análisis bivariado que, utilizando el contraste de asociación 
de variables cualitativas convertidas en variables Dummy, ${ }^{35}$ que ofrece la prueba $\mathrm{X}^{2236}$. lo que permitió establecer una caracterización más amplia de las prácticas de responsabilidad social en las firmas a partir de la identificación de estas y su correlación con diferentes elementos como los que se enuncian a continuación:

a. Tipo de empresa (Mipymes y grandes empresas respectivamente).

b. Prácticas de RSE en donde estas se destacan.

c. Falencias en concepciones como la gestión de la innovación.

d. Fortalezas y debilidades en su gestión con 6 elementos de interés.

e. Oportunidades y amenazas con la integración con stakeholders claves de la cadena de valor como clientes y proveedores.

En consecuencia, los resultados que se obtuvieron, una vez se puso en marcha la presente investigación se exponen a continuación:

\section{RESULTADOS}

Al terminar la investigación se pudo concluir que existen varias concepciones del término responsabilidad social empresarial, así como diferentes tipos de elementos que unidos, permiten desarrollar prácticas de gestión socialmente responsable, a partir del cumplimiento de una serie de responsabilidades que van desde las económicas, legales, éticas o de transparencia y finalmente, sociales como resultado de las acciones de las tres primeras. De esta manera se pudo establecer que:

- Las grandes empresas están adelantadas en el área, porque cuentan con departamentos para verificar sus acciones y para gestionar, transmitir y desarrollar su know how sobre el tema. Esto principalmente porque es un requisito para acceder a nuevos mercados como el europeo.

- Sin embargo, son las MIPYMES las que en los mismos términos de las anteriores organizaciones, se encuentran rezagadas, razón por la cual todo accionar de la academia expresado en función a sus responsabilidades académicas, de investigación y de proyección social debe enfocarse a ellas.

- En el caso del sector público, sus actuaciones por ser revisadas por la ley se acercan al cumplimiento de las responsabilidades económicas, legales y éticas; sin embargo, por tratarse de entidades con enfoque burocrático sus mismos estamentos les dificultan lograr a cabalidad su última responsabilidad.

\footnotetext{
35 Esto principalmente porque la mayoría de variables introducidas en la investigación son variables de naturaleza categórica (o cualitativas) con dos o más categorías. Por ejemplo, rango de activos, actividad económica, certificado en norma ISO 9001:2008, etcétera. Por lo cual, para poderlas analizar en el paquete SPSS se representaron como variables Dummy o indicadoras, ya que estas variables cualitativas pueden tomar valores numéricos usualmente, cero y uno. Aunque según la necesidad pueden ampliarse a más equivalencias. Así, los dos valores significan que la observación pertenece a una o más de las categorías analizadas. Las variables Dummy o indicadoras sirven para identificar categorías o clase a las que pertenecen las observaciones.

36 Esta prueba estadística permite ser utiliza para probar la independencia de dos variables entre sí, mediante la presentación de los datos en tablas de contingencia.
} 
- Por otro lado, a nivel de la empresa privada, sus prácticas de responsabilidad presentan falencias en aspectos relacionados con:

1. Las prácticas laborales que incentivan el incremento del acoso laboral.

2. Las prácticas laborales que incentivan la presencia del techo de cristal dificultando el desarrollo de mecanismos que brinden equidad a trabajadores por sexo, raza o condición política y/o sexual.

3. Prácticas comerciales enfocadas a incrementar participación en el mercado afectando a proveedores y competidores vía prácticas comerciales prohibidas como venta a pérdida, precios predatorios y dumping social. Inclusive dumping ecológico.

4. Prácticas ligadas a transparencia en la medida que dichas firmas no hacen reportes de sostenibilidad ni siquiera ante sus shareholders, ni tampoco cuentan como mecanismos de gobierno corporativo que faciliten el desarrollo de mecanismos de autogobierno.

5. Asimismo, en lo relacionado con prácticas de mercadeo responsable es necesario que las empresas integren dentro de sus procesos de gestión conceptos como la gestión del conocimiento, la gestión de la cadena de valor y la gerencia ética.

6. Un elemento adicional tiene que ver con que a partir de lo investigado, se pudo establecer que la academia debe integrarse a la cadena de valor de la organización empresarial buscando tal y como lo propone Porter (2006), un vínculo entre la ventaja competitiva y la responsabilidad social corporativa. Enfocándose principalmente en:

a. Buscar crear vínculos con centros de investigación y universidades. (Gestión del conocimiento).

b. Desarrollar infraestructura administrativa y física eficiente dentro y fuera de la organización a la que se integra.

c. Establecer mecanismos para lograr prácticas comerciales justas y abiertas.

d. Establecer mecanismos que protejan la propiedad industrial y la propiedad intelectual.

e. Pugnar por el desarrollo de prácticas asociadas a la gestión de libro abierto como mecanismo para el desarrollo de prácticas transparentes.

f. Desarrollar, desde la academia, mecanismos que permitan crear dispositivos meritocráticos de selección, contratación e incentivos para los trabajadores.

g. Involucrar en los diseños pedagógicos conceptos como la SCM, el CRM, el MRP, el TOC y el JIT entre otros como mecanismos para integrar a la gestión de la RSC a los proveedores y los consumidores de la organización empresarial.

h. El integrar a los proceso de formación organizaciones de apoyo que faciliten la gestión de estándares regulatorios que condicionan la competitividad de la firma y su acceso y sostenibilidad en nuevos mercados. 


\section{CONCLUSIONES}

En términos generales, a lo largo de la realización de esta ponencia se ha tratado de establecer que existen algunos mecanismos para caracterizar prácticas de responsabilidad social y el impacto de las mismas en el quehacer de las firmas. Igualmente, han quedado claras varias cosas como las que se mencionan a continuación:

En términos generales tienen más posibilidad de desarrollar mecanismos que faciliten la gestión de las prácticas de RSE, aquellas organizaciones con capacidad directiva enfocada en lograr dicha meta.

Sin embargo, existe un grupo de firmas sobre el cual el desarrollo de programas de formación en el tema es casi inexistente, sea por desconocimiento o por falta de interés de estos; razón por la cual, la academia en convenio con los sectores públicos y privados, deberá poner especial atención en ellos.

Así, dicho grupo estratégico está conformado por todo aquel conjunto de organizaciones que son caracterizadas por la ley 905 de 2004 y que en su artículo 2 las categoriza como:
a. Microempresa o Famiempresa
b. Pequeña empresa
c. Mediana empresa

De esta manera, como tal grupo representa en gran medida a cerca del $95 \%$ de las empresas legalmente constituidas, es necesario considerar que sobre ellas deberá girar en gran medida la política de formación, capacitación y gestión del conocimiento del país. Es por esta circunstancia, que a raíz de lo que se ha visto en esta investigación, se deberá considerar que cualquier programa de formación que se quiera desarrollar para mejorar, desarrollar y gestionar prácticas de RSE deberá tener en cuenta elementos como los siguientes:

a. Gobierno empresarial, enfocándose en ofrecer información y herramientas a las MIPYMES que deseen establecer relaciones con sus Shareholders (accionistas) y demás Stakeholders, enfocándose en la mejora de sus prácticas de gobierno empresarial, haciendo énfasis en conceptos como: dirección estratégica, ética, gestión de los Stakeholders, transparencia, derechos y deberes de los Shareholders, integridad y demás elementos asociados a la ley, la moral y las buenas costumbres mercantiles.

b. En lo relacionado con Prácticas Laborales, cualquier programa de formación que se desarrolle deberá enfocarse en medir las relaciones que ostenta ésta con sus trabajadores, buscando mejorar sus prácticas laborales en parámetros como: derechos laborales, salud ocupacional, seguridad laboral y relaciones industriales. 
c. Respecto a la Gestión Medioambiental, será necesario establecer a partir de lo citado en la presente investigación, programas de formación que hagan énfasis en temas como el uso eficiente de los recursos, la prevención y mitigación de impactos asociados a la contaminación (residuos, emisiones y vertidos), así como también en el desarrollo y la utilización de métodos y tecnologías ambiental y socialmente sostenibles.

d. En lo que tiene que ver con aprovisionamiento y cadena de valor, es necesario capacitar a la MIPYME en todo lo relacionado con la gestión de las relaciones con sus proveedores, a través de la mejora continua de procesos asociados a la logística, evaluación y selección de proveedores y desarrollo y certificación de proveedores, buscando así desarrollar mecanismos de aprendizaje de doble nivel.

e. En cuanto a Mercadotecnia, es necesario ofrecer mecanismos de información que le permita a la firma enfocar las relaciones con sus clientes a través de la mejora de sus prácticas de mercadeo en temas como marketing social, negocios inclusivos, marketing reputacional, branding social, y gestión de productos y servicios desde la logística inversa. ${ }^{37}$

f. Mecanismos de Inversión Social: este acápite deberá tener en cuenta mecanismos asociados con la gestión del riesgo, la evaluación del portafolio, la cobertura de necesidades de la comunidad buscando medir la rentabilidad en función a la medición de impactos de la cadena de valor, las dimensiones sociales del contexto competitivo, y los temas sociales genéricos asociados al resultado de dichas inversiones.

37 Otro acápite a tomar en cuenta en este tema tiene que ver con prácticas comerciales negativas como la venta piramidal, el precio predatorio, la venta a pérdida y el dumping social que se pueden dar muchas veces independiente de si la empresa tiene o no posición de dominio en el mercado. 
Se espera que a futuro la academia colombiana pueda entrar a desarrollar su propia versión del índice NASCO,${ }^{38}$ con el fin de entrar en colaboración con la sociedad y el sector real para que puedan desarrollar modelos de gestión sostenibles, empresarialmente competitivos y social y ambientalmente sustentables en el tiempo.

\section{REFERENCIAS BIBLIOGRÁFICAS}

ASOCIACIÓN NACIONAL DE INDUSTRIALES, ANDI. (2009). Encuesta de responsabilidad social. [En línea], disponible: http://www.andi.com.co/pages/proyectos paginas/proyectos_detail.aspx?pro_id $=69 \& \mathrm{Id}=6 \&$ clase $=8 \&$ Tipo $=3$, recuperado: $27 \mathrm{de}$ marzo de 2010.

CARROLL, A. (1979), A Three Dimensional Conceptual Model of Corporate Performance. Academy of Management Review, 4 (4), pgs. 497-505.

CARROLL, A. (1999), Corporate Social Responsibility. Business and society, 38 (3), 268-295.

FREEMAN, R.E. (1984), Strategic Management: A Stakeholder Approach. Pitman Series in Business and Public Policy.

GLOBAL REPORTING INITIATIVA. (2006), Guía para la elaboración de memorias de sostenibilidad: versión G3. [En línea], disponible en: http://www.globalreporting.org/ ReportingFramework/ G3Online/, recuperado: 27 de Marzo de 2010.

38 El proyecto NASCO es una medida del Artículo 6 del Fondo Social Europeo (FSE), iniciado en noviembre de 2001 que nace con objeto de aumentar la Responsabilidad Social de las Empresas (RSE) implicando a las PYMES en actividades de investigación práctica. Su esencia de operación se enfoca en concienciar a pequeñas y medianas empresas de que existen muchas formas de actuación que pueden adoptar en sus actividades empresariales. El reconocimiento de una interrelación entre empresas y sociedad permitiría un desarrollo más sostenible y satisfacer en mayor medida las expectativas de todas las partes interesadas.

Los objetivos de NASCO reflejan la voluntad manifestada en el Green Paper for Promoting a European Framework for RSE, siendo éstos principalmente, los siguientes:

- Difundir la responsabilidad Social de las Empresas ampliando el número de usuarios de herramientas de gestión que contemplen la acción social.

- Difundir documentos específicos sobre Buenas Prácticas en materia de RSE.

- Implicar a PYMES de diferentes países europeos en la aplicación de herramientas que les permitan conocer su grado de implicación en relación a sus actividades para con la sociedad.

- Planificar y desarrollar un modelo de gestión que facilite una amplia adopción del Balance Social como elemento evaluador de los aspectos sociales en las actividades de las empresas.

Los resultados esperados derivados de la actuación del NASCO son:

- Creación y difusión de documentos relevantes y referentes sobre Buenas Prácticas de gestión en materia de RSE.

- Creación y consolidación de una Base de Datos europea de las empresas implicadas en cuestiones de responsabilidad Social.

- Creación de documentación dirigida a la aplicación del balance Social en las empresas.

- Desarrollo de un proceso de consulta y formación para la adopción del Balance Social en las PYMES.

- Estandarización de un modelo de Balance Social reproducible.

Disponible en: http://www.euquality.com/zonaprivada/descargas/Octubre\%202007\%20-\%20Indice\%20Nasco\%20I., recuperado: 10 de Enero de 2010. 
ÍNDICE NASCO. (2003), Una herramienta para mostrar y medir la responsabilidad social en las PYMES. Unión Europea, Fondo Social Europeo, artículo 6, medidas innovadoras, versión número 1.

PORTER, M., Y KRAMER, M. (2006), Estrategia y sociedad: El vínculo entre ventaja competitiva y responsabilidad social corporativa. Harvard Business Review América Latina, diciembre.

REYES, A.A. (2009), Presentación del curso de profundización en Gerencia Estratégica Responsable. Auditorio de la Universidad Nacional Abierta y a distancia (UNAD). Bogotá. D.C, Colombia. 